\title{
An Energy-Efficient Handover Scheme with Geographic Mobility Awareness in WiMAX-WiFi Integrated Networks
}

\author{
Wen-Hsin Yang*†, You-Chiun Wang*, Yu-Chee Tseng* \\ * Department of Computer Science \\ National Chiao-Tung University \\ Hsinchu, Taiwan 30010, R.O.C. \\ Email: $\{$ wenhsin, wangyc, yctseng $@ @$ cs.nctu.edu.tw
}

\begin{abstract}
WiFi networks have been deployed in many regions such as buildings and campuses to provide wireless Internet access. However, to support ubiquitous wireless service, one possibility is to integrate these narrow-range WiFi networks with a wide-range network such as WiMAX. Under this WiMAX-WiFi integrated network, how to conduct energy-efficient handovers is a critical issue. In this paper, we propose a handover scheme with geographic mobility awareness (HGMA) by considering the past handover patterns of mobile devices. HGMA can conserve the energy of handovering devices from three aspects. First, it prevents mobile devices from triggering unnecessary handovers by measuring their received signal strength and moving speeds. Second, it includes a handover candidate selection (HCS) method for mobile devices to intelligently select a subset of WiFi access points or WiMAX relay stations to be scanned. Therefore, mobile devices can reduce their network scanning and thus save their energy. Third, HGMA prefers mobile devices staying in their original WiMAX or WiFi networks. This can prevent devices from consuming too much energy on interface switching. Simulation results show that HGMA can reduce about $69 \%$ and $30 \%$ of energy consumption on network scanning and interface switching, respectively, and with $16 \%$ to $64 \%$ more probabilities for mobile devices staying in WiFi networks.
\end{abstract}

Index Terms-energy efficiency, handover, network selection, WiFi, WiMAX, wireless network.

\section{INTRODUCTION}

Recently, various multimedia applications such as video streaming and VoIP services have become popular and many smart handheld devices have been ready to embrace these services. Meanwhile, in many buildings and campuses, WiFi networks have been deployed to support broadband wireless access. However, these WiFi networks can only cover small geographic regions. To support seamless wireless access, the emerging WiMAX network [1], [2] is considered to be able to provide global coverage. In this paper, we consider such a WiMAX-WiFi integrated network that is operated by the same service provider, as shown in Fig. 1. WiMAX network serves as a wireless backbone with relay capability and each WiFi network is connected to the former to provide wireless Internet access. Mobile devices (MDs) are equipped with WiMAXWiFi dual interfaces. However, an MD only needs to keep one connection through one of its interfaces at a time.

\author{
Bao-Shuh P. Lin*† \\ $\dagger$ Information and Communications Research Laboratories \\ Industrial Technology Research Institute \\ Chutung, Hsinchu, Taiwan 31040, R.O.C.
Email: bplin@itri.org.tw \\ Chutung, Hsinchu, Taiwan 31040,
Email: bplin@itri.org.tw
}

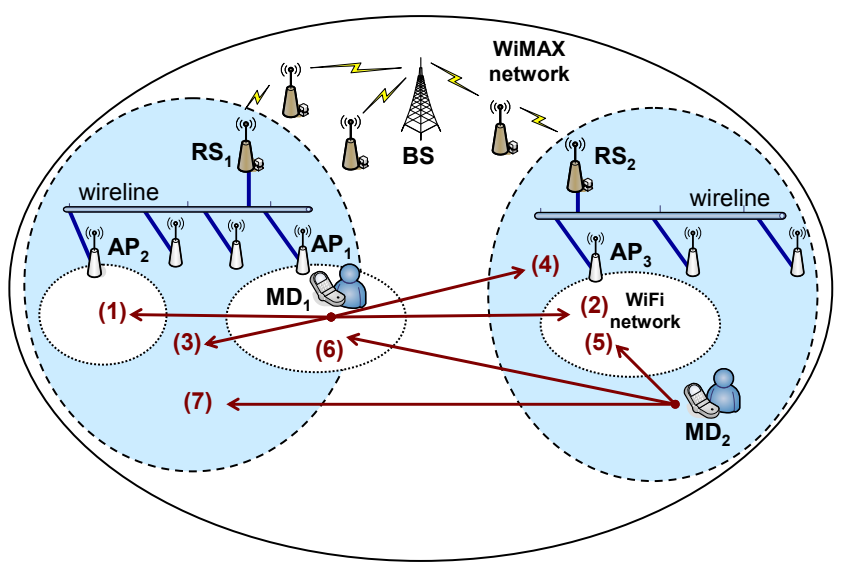

Fig. 1: A WiMAX-WiFi integrated network.

Under this WiMAX-WiFi integrated network, how to conduct energy-efficient handovers is a critical issue. Since WiFi networks are often deployed to cover certain indoor regions such as offices or outdoor regions such as campuses, users would be expected to move following some geographic patterns. Therefore, MDs would move and handover in some predictable ways, and we call this as the geographic mobility (GM) feature of MDs. References [3], [4], [5] indicate that such a GM feature has significant effects on designing a handover scheme. Actually, it can help conserve MDs' energy because we can predict potential handover targets. In this paper, we propose a handover scheme with geographic mobility awareness (HGMA), which can conserve MDs' energy from three aspects. First, it prevents MDs from conducting too frequent handovers in a short duration (called ping-pong effect). HGMA will measure the moving speeds and received signal strength (RSS) of MDs to avoid unnecessary handovers. Second, we develop a handover candidate selection (HCS) method for MDs to intelligently select a subset of WiFi access points (APs) or WiMAX relay stations (RSs) to be scanned, according to MDs' GM features. HCS can predict the potential APs/RSs that an MD may handover to, based on the past 
handover patterns. Therefore, MDs can reduce the number of APs/RSs to be scanned and thus save their energy. Third, HGMA prefers MDs staying in their original WiMAX or WiFi networks. This can prevent MDs from frequently switching network interfaces and thus saving their energy.

In the literature, research efforts [6], [7], [8], [9], [10] have focused on how to conduct handovers between a lowbandwidth cellular network and high-bandwidth WLANs. These results may not be directly applied in our WiMAXWiFi integrated network because both WiMAX and WiFi are high-bandwidth networks. The work [11] proposes a proactive handover scheme between WLANs and a $3 \mathrm{G}$ network. It maintains a handover trigger table that records the locations where APs' signals will drop significantly. This work requires the knowledge of MDs' positions to decide whether it should trigger pre-handoff operations. Several studies consider the handover issue in a WiMAX-WiFi integrated network. The work [12] avoids unnecessary handovers in an IEEE 802.16aIEEE $802.11 \mathrm{n}$ integrated network by detecting the changes of RSS. It prefers keeping MDs in the 802.11n network to get a higher bandwidth. Reference [13] proposes a bandwidthbased fuzzy handover scheme in an 802.16-802.11 integrated network. However, both [12] and [13] do not address the energy issue. The work [14] considers that WiMAX base stations $(B S s)$ will periodically broadcast the density of WiFi APs within their coverages. Such information can help MDs to adjust their active scan interval to search for APs to reduce their energy consumption. This is different from our approach because we focus on reducing scans in both directions.

Our major contributions are twofold. First, we identify the GM feature of MDs in a WiMAX-WiFi integrated network. Second, we propose an energy-efficient HGMA handover scheme by adopting the GM feature. Our HGMA scheme can eliminate unnecessary handovers, reduce the number of network scanning, and avoid switching network interfaces too frequently. Extensive simulation results show that HGMA can save about $69 \%$ of network scanning, reduce about $30 \%$ of interface switching, and keep MDs in WiFi networks with $16 \%$ to $64 \%$ more probabilities.

The rest of this paper is organized as follows: Section II presents the system architecture. Section III proposes our handover schemes. Section IV gives our simulation results. Conclusions are drawn in Section V.

\section{WiMAX-WiFi Integrated Network ARchitectUe}

The system architecture of a WiMAX-WiFi integrated network has two tiers, as shown in Fig. 1. The upper WiMAX tier contains a BS connecting to multiple RSs. On the lower WiFi tier, each RS is connected to multiple APs through wirelines. We say that an RS and an AP have a parent-child relationship if they are connected through a wireline. The wireless coverage of adjacent APs are not necessarily overlapped with each other. We assume that the whole service area are completely covered by the WiMAX network, but may not be completely covered by the WiFi networks. We assume that both these WiMAX and WiFi networks belong to the same service provider. Each
MD is equipped with dual $802.11 \mathrm{~b} / \mathrm{g}$ and $802.16 \mathrm{e}$ interfaces, but it only needs to turn on one interface at at time.

There are seven handover cases in the WiMAX-WiFi integrated network, as numbered by 1-7 in Fig. 1:

1) $\mathrm{MD}_{1}$ handovers from $\mathrm{AP}_{1}$ to $\mathrm{AP}_{2} . \mathrm{AP}_{1}$ and $\mathrm{AP}_{2}$ have the same parent $\mathrm{RS}_{1}$.

2) $\mathrm{MD}_{1}$ handovers from $\mathrm{AP}_{1}$ to $\mathrm{AP}_{3} . \mathrm{AP}_{1}$ and $\mathrm{AP}_{3}$ have different parent RSs.

3) $M D_{1}$ handovers from $A P_{1}$ to its parent $R S_{1}$.

4) $\mathrm{MD}_{1}$ handovers from $A P_{1}$ to another $R S_{2}$.

5) $\mathrm{MD}_{2}$ handovers from $\mathrm{RS}_{2}$ to its child $\mathrm{AP}_{3}$.

6) $\mathrm{MD}_{2}$ handovers from $\mathrm{RS}_{2}$ to another $\mathrm{AP}_{1}$.

7) $\mathrm{MD}_{2}$ handovers from $\mathrm{RS}_{2}$ to $\mathrm{RS}_{1}$.

In cases 3 and 4, the MD has to switch to the WiMAX mode. In cases 5 and 6 , the MD can switch to the WiFi mode to save energy. The above cases can be categorized into two classes. The $H O_{A P}$ class contains handover cases $1-4$, where an MD moves out of its current AP. The $H O_{R S}$ class contains handover cases 5-7, where an MD moves out of its current RS. Table I summarizes our notations.

\section{THE HGMA SCHEME}

Fig. 2 shows our HGMA scheme. It has two parts, namely $\mathrm{HO}_{\mathrm{AP}}$ and $\mathrm{HO}_{\mathrm{RS}}$ classes. When an MD is currently associated with an $\mathrm{AP}_{i}$ (i.e., $\mathrm{HO}_{\mathrm{AP}}$ class), it periodically checks whether it should trigger a handover. The checking procedure will be discussed in Section III-A. If triggering a handover is needed, it will invoke the HCS method in Section III-B to select a set of candidate APs, $\mathcal{C}_{\mathrm{AP}}$. The MD then scans those APs in $\mathcal{C}_{\mathrm{AP}}$ and calculates each AP's available bandwidth (for example, the results in [15], [16] point out some ways to calculate an AP's remaining bandwidth). Then, among those APs whose available bandwidths can afford the MD's traffic demand $B_{\mathrm{MD}}$, the MD will select the AP with the strongest RSS to handover to.

If the $\mathrm{MD}$ fails to find an available $\mathrm{AP}$ in $\mathcal{C}_{\mathrm{AP}}$, it will invoke the HCS method in Section III-B, which will return a set of candidate RSs, $\mathcal{C}_{\mathrm{RS}}$. Then, it scans these RSs and query their available bandwidths by a request-grant protocol. Among those RSs whose available bandwidths can afford the bandwidth demand $B_{\mathrm{MD}}$, the MD will select the RS with the strongest RSS to handover to. However, if no feasible RS can be found, the MD will search other APs/RSs (not in $\mathcal{C}_{\mathrm{AP}} / \mathcal{C}_{\mathrm{RS}}$ ).

On the other hand, when an MD is currently associated with an $\mathrm{RS}_{i}$ (i.e., $\mathrm{HO}_{\mathrm{RS}}$ class), it periodically checks whether it should trigger a handover. If so, the MD will invoke the HCS method and then scan the candidate RSs in $\mathcal{C}_{\mathrm{RS}}$. If this step fails (case I in Fig. 2), it will check whether its current energy level $e_{\mathrm{MD}}$ is larger than a predefined threshold $\varepsilon_{\mathrm{RS}}$. If so, the MD will invoke the HCS method, turn on its WiFi interface, scan the candidate APs in $\mathcal{C}_{\mathrm{AP}}$, find one target $\mathrm{AP}$, and add the selected AP into its $\mathcal{L}_{\mathrm{AP}}\left(R S_{i}\right)$, that records all APs to which the MD has ever handovered from its current $R S_{i}$. In the above discussion, even if the MD decides not to trigger handover (see case II in Fig. 2), it will still try to search for APs if both $\mathcal{L}_{\mathrm{AP}}\left(R S_{i}\right) \neq \emptyset$ (that is, there are potential 
TABLE I: Summary of notations.

\begin{tabular}{ll}
\hline notation & definition \\
\hline$e_{\mathrm{MD}}$ & current energy level of an MD $(0$ to $\gamma$, empty to fully-charged $)$ \\
$\varepsilon_{\mathrm{RS}}$ & energy threshold to switch to the WiFi interface in the HO $\mathrm{RS}_{\mathrm{RS}}$ class \\
$V$ & current speed of an MD \\
$B_{\mathrm{MD}}$ & bandwidth demand of an MD \\
$\Delta_{\mathrm{MD}}$ & dwell-time of an MD \\
$V_{0}(A P) / V_{0}(R S)$ & average speed of an MD when it is within a WiFi/WiMAX network \\
$T_{A P}(V) / T_{R S}(V)$ & average observation period for an MD to measure RSS in a WiFi/WiMAX network \\
$n_{\mathrm{exp}}$ & expected number of candidate APs/RSs \\
$\mathcal{L}_{\mathrm{AP}}\left(R S_{i}\right)$ & table in an MD that records the neighboring APs of the associating RS \\
$\mathcal{C}_{\mathrm{AP}} / \mathcal{C}_{\mathrm{RS}}$ & candidate APs/RSs returned by HCS \\
$\mathcal{T}_{\mathrm{AP}}\left(A_{i}\right) / \mathcal{T}_{\mathrm{RS}}\left(A_{i}\right)$ & table that records neighboring APs/RSs of an AP $A_{i}$ \\
$\mathcal{T}_{\mathrm{AP}}\left(R_{i}\right) / \mathcal{T}_{\mathrm{RS}}\left(R_{i}\right)$ & table that records neighboring APs/RSs of an RS $R_{i}$ \\
\hline
\end{tabular}

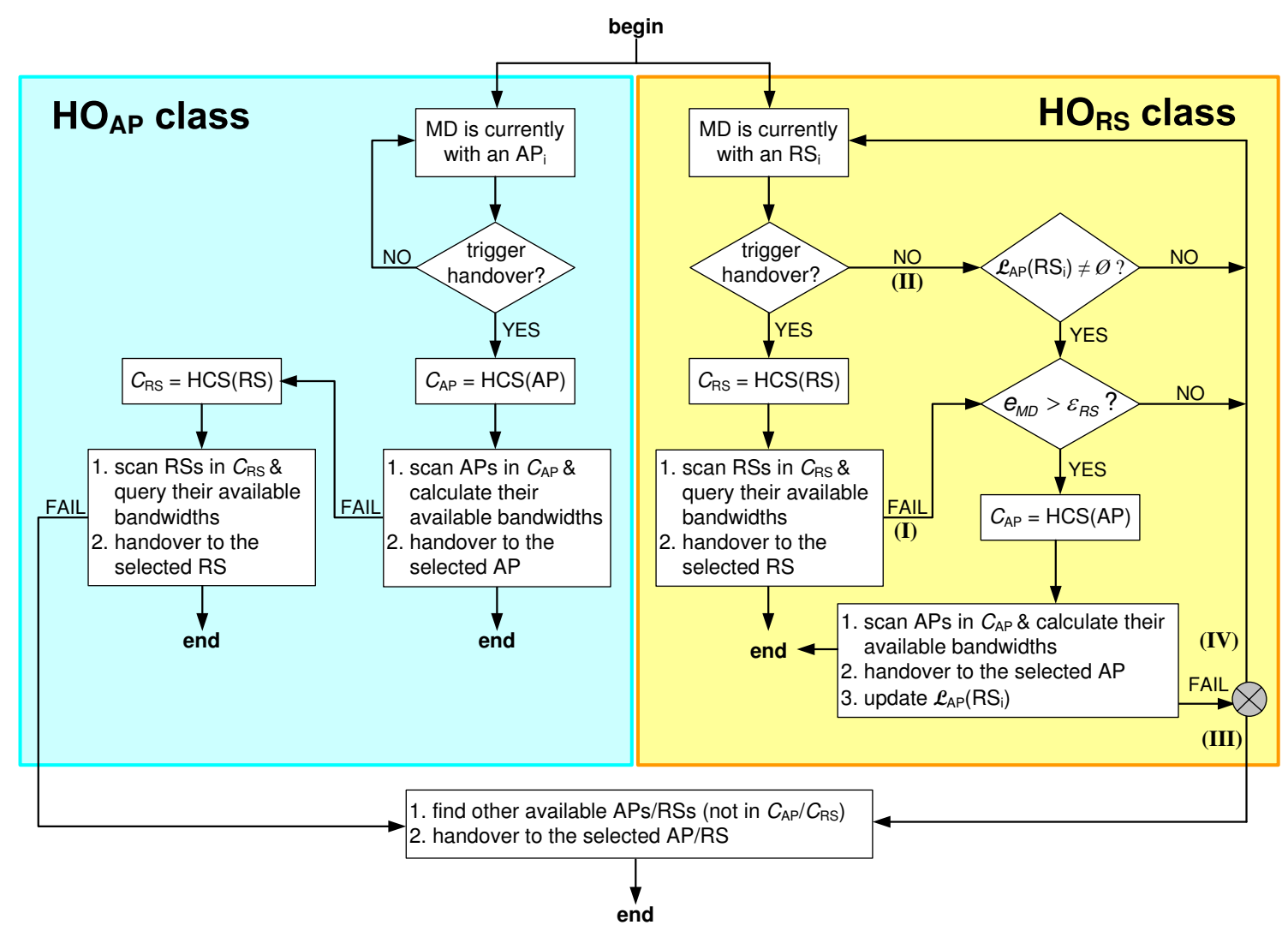

Fig. 2: The HGMA scheme.

APs in this $R S_{i}$ 's neighborhood) and $e_{\mathrm{MD}}>\varepsilon_{\mathrm{RS}}$ (that is, the MD has sufficient energy to conduct extra network scanning). In this case, the MD will invoke the HCS method, scan the candidate APs in $\mathcal{C}_{\mathrm{AP}}$, find one target $\mathrm{AP}$, and add the selected AP into its $\mathcal{L}_{\mathrm{AP}}\left(R S_{i}\right)$. However, when the MD cannot find any feasible RS/AP, two cases will happen (refer to the switch point ' $\bigotimes$ ' in Fig. 2). If the MD arrives at $\otimes$ from case I, it will follow case III and search other APs/RSs not in $\mathcal{C}_{\mathrm{AP}} / \mathcal{C}_{\mathrm{RS}}$. However, if the MD arrives at $\otimes$ from case II, it will follow case IV and keep associating with its current RS.

\section{A. When to Trigger a Handover}

We then discuss when to trigger a handover event. In the $\mathrm{HO}_{\mathrm{AP}}$ class, the MD will periodically measure the average
RSS from its associating AP. Typically, a low RSS will trigger an MD to start a handover process. To alleviate the pingpong effect when an MD is around cell boundaries due to temporal RSS dropping, we propose adaptively adjusting the observation period to $T_{A P}(V)=\frac{V_{0}(A P)}{V} \times T_{0}(A P)$, where $V_{0}(A P)$ is the average speed of the MD when it is within WiFi networks, $V$ is the MD's current speed, and $T_{0}(A P)$ is a constant representing the MD's average observation period. When the average RSS is below the handover threshold for a period of $T_{A P}(V)$ time, it means that the MD is very close to the coverage boundary of that AP. Thus, the MD has to trigger a handover event. Similarly, in the $\mathrm{HO}_{\mathrm{RS}}$ class, the MD will continuously measure the average RSS from its associating RS. When the RSS is below the handover threshold for a 
period of $T_{R S}(V)=\frac{V_{0}(R S)}{V} \times T_{0}(R S)$ time, a handover event should be triggered.

\section{B. The HCS Method}

In HGMA, the HCS method will be invoked to select the candidate $\mathrm{APs} / \mathrm{RSs}$ that an MD is more likely to handover to. HCS exploits the GM feature based on past handover patterns. Specifically, for each AP $A_{i}$, we maintain two tables $\mathcal{T}_{\mathrm{AP}}\left(A_{i}\right)$ and $\mathcal{T}_{\mathrm{RS}}\left(A_{i}\right)$ to record the information of $A_{i}$ 's neighboring APs and RSs, respectively. An AP $A_{j}$ is in $A_{i}$ 's $\mathcal{T}_{\mathrm{AP}}\left(A_{i}\right)$ if $A_{i}$ and $A_{j}$ share the same parent RS or an MD has ever handovered from $A_{i}$ to $A_{j}$. An RS is in $A_{i}$ 's $\mathcal{T}_{\mathrm{RS}}\left(A_{i}\right)$ if it is $A_{i}$ 's parent RS or an MD has ever handovered from $A_{i}$ to the RS. Similarly, for each RS $R_{i}$, we maintain its neighboring RSs and APs in tables $\mathcal{T}_{\mathrm{RS}}\left(R_{i}\right)$ and $\mathcal{T}_{\mathrm{AP}}\left(R_{i}\right)$, respectively. An RS $R_{j}$ is in $R_{i}$ 's $\mathcal{T}_{\mathrm{RS}}\left(R_{i}\right)$ if an MD has ever handovered from $R_{i}$ to $R_{j}$. An AP is in $R_{i}$ 's $\mathcal{T}_{\mathrm{AP}}\left(R_{i}\right)$ if it is a child $\mathrm{AP}$ of $R_{i}$ or an MD has ever handovered from $R_{i}$ to it.

In the above four tables, two extra pieces of information are maintained. Without loss of generality, we consider table $\mathcal{T}_{\mathrm{AP}}\left(A_{i}\right)$ of an $\mathrm{AP} A_{i}$. For each $A_{j}$ in $\mathcal{T}_{\mathrm{AP}}\left(A_{i}\right)$, we record two statistics: $v\left(A_{i}, A_{j}\right)$ counts the total number of MDs that have ever handovered from $A_{i}$ to $A_{j}$ and $t\left(A_{i}, A_{j}\right)$ records the average dwell-time in $A_{i}$ for these MDs. The other three tables $\mathcal{T}_{\mathrm{RS}}\left(A_{i}\right), \mathcal{T}_{\mathrm{AP}}\left(R_{i}\right)$, and $\mathcal{T}_{\mathrm{RS}}\left(R_{i}\right)$ also maintain the similar information.

HCS works as follows: Without loss of generality, we consider an MD that currently associates with an AP $A_{i}$ and intends to scan other APs. HCS will select a number $n=$ $\min \left\{n_{\exp },\left|\mathcal{T}_{\mathrm{AP}}\left(A_{i}\right)\right|\right\}$ of candidate APs from its $\mathcal{T}_{\mathrm{AP}}\left(A_{i}\right)$ to be scanned, where $n_{\text {exp }}$ is the expected number of candidate APs and $\left|\mathcal{T}_{\mathrm{AP}}\left(A_{i}\right)\right|$ is the total number of entries in $\mathcal{T}_{\mathrm{AP}}\left(A_{i}\right)$. One way to define $n_{\exp }$ is to consider the MD's remaining energy. When the MD has more energy, a larger $n_{\exp }$ can be used. To select these $n$ APs, we calculate the similarity $s\left(A_{i}, A_{j}\right)$ of $A_{i}$ and $A_{j}$ for each $A_{j} \in \mathcal{T}_{\mathrm{AP}}\left(A_{i}\right)$ as follows:

$$
s\left(A_{i}, A_{j}\right)=\sqrt{W_{v} \times f_{v}\left(A_{i}, A_{j}\right)^{2}+W_{t} \times f_{t}\left(A_{i}, A_{j}\right)^{2}},
$$

where $0<W_{v}, W_{t}<1, W_{v}+W_{t}=1$, and

$$
\begin{aligned}
f_{v}\left(A_{i}, A_{j}\right) & =\frac{v\left(A_{i}, A_{j}\right)}{\sum_{A_{k} \in \mathcal{T}_{\mathrm{AP}}\left(A_{i}\right)} v\left(A_{i}, A_{k}\right)}, \\
f_{t}\left(A_{i}, A_{j}\right) & =\frac{\min \left\{\Delta_{\mathrm{MD}} t\left(A_{i}, A_{j}\right)\right\}}{\max \left\{\Delta_{\mathrm{MD}}, t\left(A_{i}, A_{j}\right)\right\}},
\end{aligned}
$$

where $\Delta_{\mathrm{MD}}$ is the current dwell-time that the MD has stayed in $A_{i}$. Then, HCS selects the first $n$ most similar APs in $\mathcal{T}_{\mathrm{AP}}\left(A_{i}\right)$ and stores them in $\mathcal{C}_{\mathrm{AP}}$. In Eq. (1), an $A_{j}$ has a higher similarity with $A_{i}$ if the visiting frequency $f_{v}\left(A_{i}, A_{j}\right)$ is higher (refer to Eq. (2)). Also, an $A_{j}$ has a higher similarity with $A_{i}$ if the dwell-time relationship $f_{t}\left(A_{i}, A_{j}\right)$ is higher (refer to Eq. (3)). When the MD's dwell-time $\Delta_{\mathrm{MD}}$ is close to $t\left(A_{i}, A_{j}\right)$, it means that the MD may follow the past handover pattern and handover to $A_{j}$ with a higher probability. We use two weights $W_{v}$ and $W_{t}$ to normalize these two factors.

\section{EXPERIMENTAL RESULTS}

In this section, we present some simulation results to verify the effectiveness of the proposed HGMA scheme. Our simulations are conducted by the IEEE 802.16 modules based on ns-2 [17]. The physical layer adopts an OFDM (orthogonal frequency division multiplexing) module and the radio propagation model is set as two-ray ground. For WiFi networks, we consider an association failure probability $p=0.2 \sim 0.4$. When an AP suffers from a higher $p$ (due to reasons such as contention), it will cause MDs to associate with it with a lower probability. We consider two types of network topologies. In the dense topology, each RS has 10 child APs. In the random topology, each RS has arbitrarily 0 to 10 child APs. Each MD will consume $5 \%$ of its energy every five minutes. We rank an MD's remaining energy by levels. When fully charged, the energy level is $\gamma$, where $\gamma \in \mathbb{N}$. The calculation of energy level is as follows:

$$
\left\lfloor\frac{\text { current remaining energy }}{\text { fully-charged energy }} \times \gamma\right\rfloor \text {. }
$$

We set $\varepsilon_{\mathrm{RS}}=1$ and $W_{v}=W_{t}=\frac{1}{2}$.

The BonnMotion tool [18] is adopted to generate two types of mobility models of MDs. In the random waypoint model, an MD randomly chooses one destination to move to, with an average speed of $[0,1] \mathrm{m} / \mathrm{s}$. When the MD reaches its destination, it pauses approximately 120 seconds and then selects another destination to move to. In the Manhattan grid model, MDs move on a number of horizontal and vertical streets in an urban area. The speeds of MDs range from 0.5 to $1 \mathrm{~m} / \mathrm{s}$, with a maximum pause time of 120 seconds. Each MD may change its direction when it reaches an intersection, with a turning probability of 0.5 . In our simulations, we set $V_{0}(A P)=0.5 \mathrm{~m} / \mathrm{s}, V_{0}(R S)=1 \mathrm{~m} / \mathrm{s}$, and $T_{0}(A P)=$ $T_{0}(R S)=100 \mu \mathrm{s}$. We mainly compare our HGMA scheme with the traditional handover scheme, where a handovering MD will scan all APs/RSs around it.

\section{A. Number of Network Scanning}

Fig. 3 shows the average number of APs scanned by MDs. Clearly, the average number of APs scanned by MDs in the dense topology is larger than that in the random topology because the former has a lager AP density. The traditional handover scheme will ask MDs to scan all possible APs around them, even though they have low remaining energy. On the contrary, HGMA allows MDs to scan less APs when they have low energy. In this way, the energy of MDs can be conserved. When comparing these two mobility models, we can observe that HGMA will cause more scans in the random waypoint model than in the Manhattan grid model when there are more than $45 \%$ of remaining energy. This is because the random waypoint model has a less regular mobility pattern, causing lower predictability, and a higher $n_{\exp }$ value is used. With the Manhattan grid pattern, the candidate APs/RSs are more predictable.

Table II summarizes the average reduction of the number of APs scanned by MDs in our HGMA scheme. We can observe 

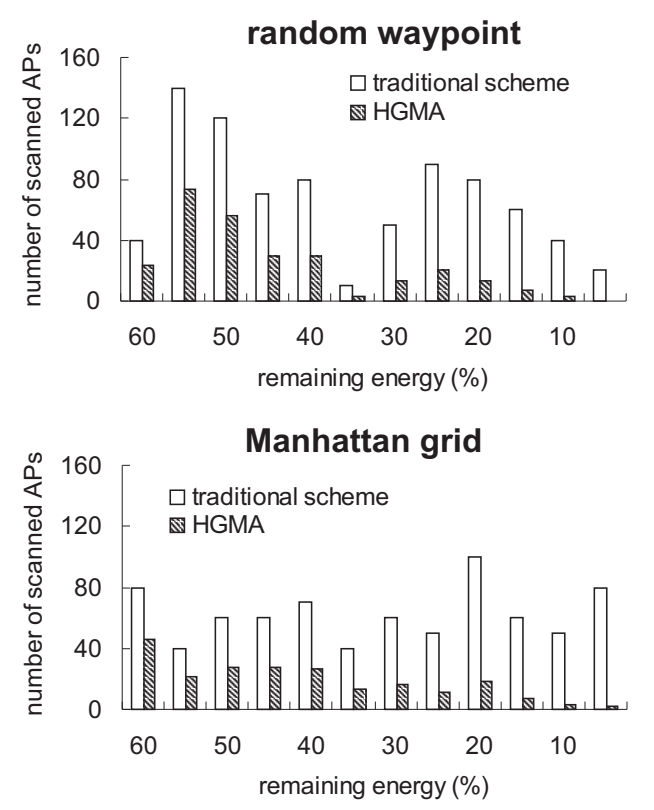

(a) dense topology
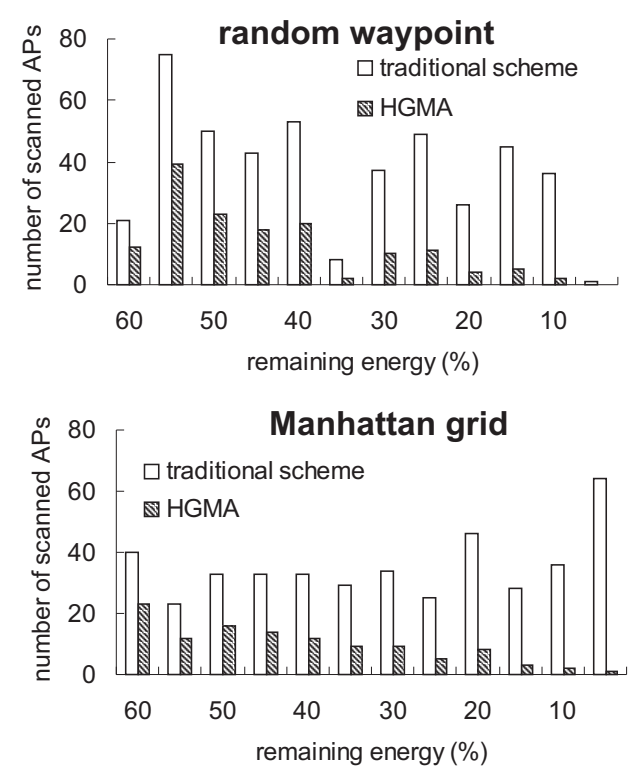

(b) random topology

Fig. 3: Average number of APs scanned by MDs.

that HGMA can reduce about $69 \%$ APs to be scanned, thereby significantly reducing the energy consumption of MDs.

TABLE II: Reduction of the number of APs scanned by MDs in HGMA.

\begin{tabular}{l|ll|c}
\hline $\begin{array}{l}\text { network } \\
\text { topology }\end{array}$ & $\begin{array}{l}\text { random } \\
\text { waypoint }\end{array}$ & $\begin{array}{l}\text { Manhattan } \\
\text { grid }\end{array}$ & average \\
\hline dense & $66.13 \%$ & $70.80 \%$ & $68.47 \%$ \\
random & $67.12 \%$ & $73.11 \%$ & $70.12 \%$ \\
\hline
\end{tabular}
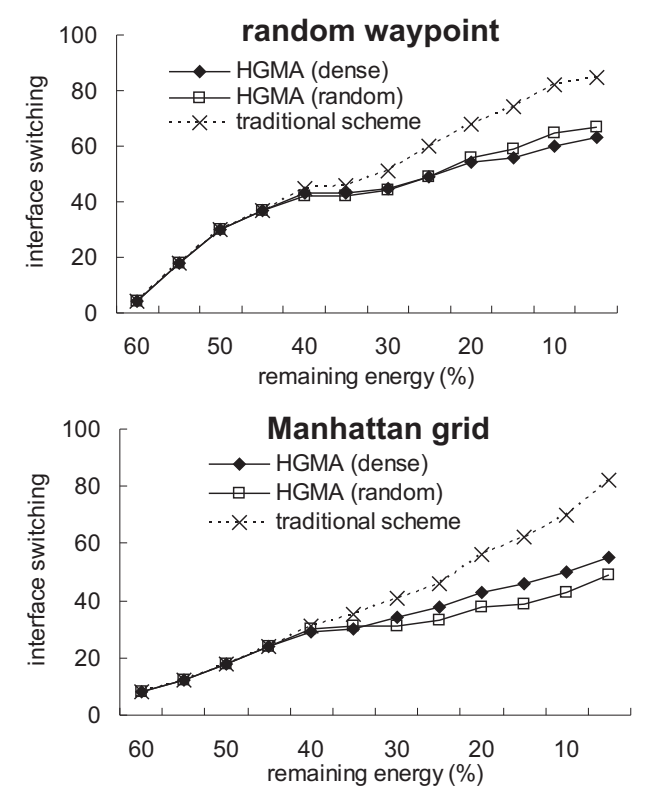

Fig. 4: Cumulative number of interface switching.

\section{B. Cumulative Number of Interface Switching}

Fig. 4 shows the cumulative number of interface switching of MDs. When MDs have more than $40 \%$ of remaining energy, HGMA has almost no effect on interface switching. This is because it encourages those MDs associating with RSs to handover to WiFi networks when they have more energy. However, when MDs have less remaining energy, HGMA will prevent them from frequently switching network interfaces. From Fig. 4, we can observe that HGMA causes the least interface switching under the Manhattan grid model due to the predictable GM feature. In average, HGMA can reduce about $30 \%$ of the number of interface switching.

\section{Cumulative Number of Handovers to APs}

Fig. 5 shows the cumulative number of handovers to APs. When the failure probability $p$ increases, the number of handovers to APs will decrease. From Fig. 5(a), we can observe that HGMA can still perform well even with a larger $p$. This is because the AP density is large enough and thus MDs can easily find an AP to handover to. On the other hand, in Fig. 5(b), the improvement of HGMA is less significant when $p$ is larger. This is because MDs may not always find suitable APs in their neighborhood. However, when MDs move in the more regular Manhattan grid model, HGMA can increase the possibility that MDs associate with WiFi networks.

Table III gives the improvement of the number of handovers to APs by HGMA. In average, HGMA can increase $64.1 \%$ and $16.1 \%$ of probability for MDs to associate with APs when $p$ is set to 0.2 and 0.4 , respectively.

\section{CONClusion}

In this paper, we have defined a WiMAX-WiFi integrated network architecture and discussed the corresponding handover scenarios. We have proposed an energy-efficient HGMA 

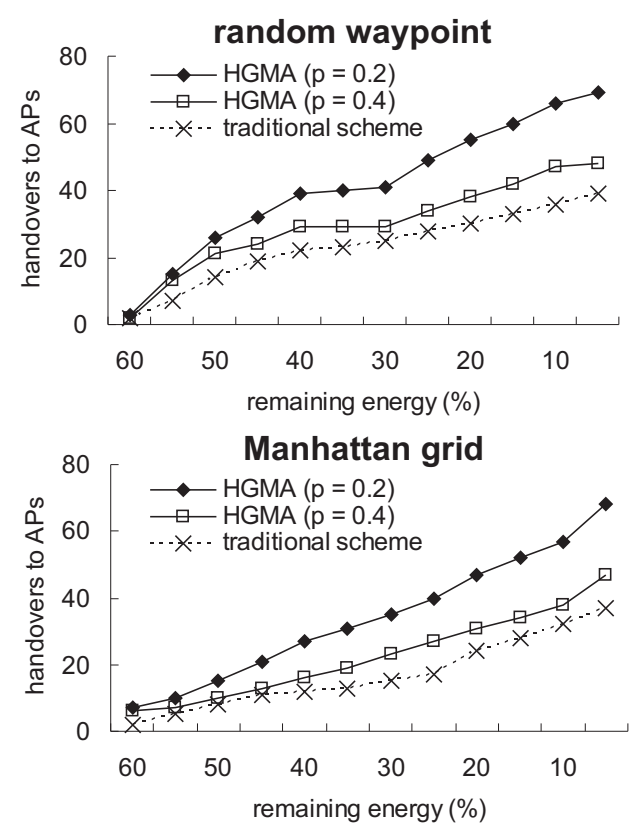

(a) dense topology
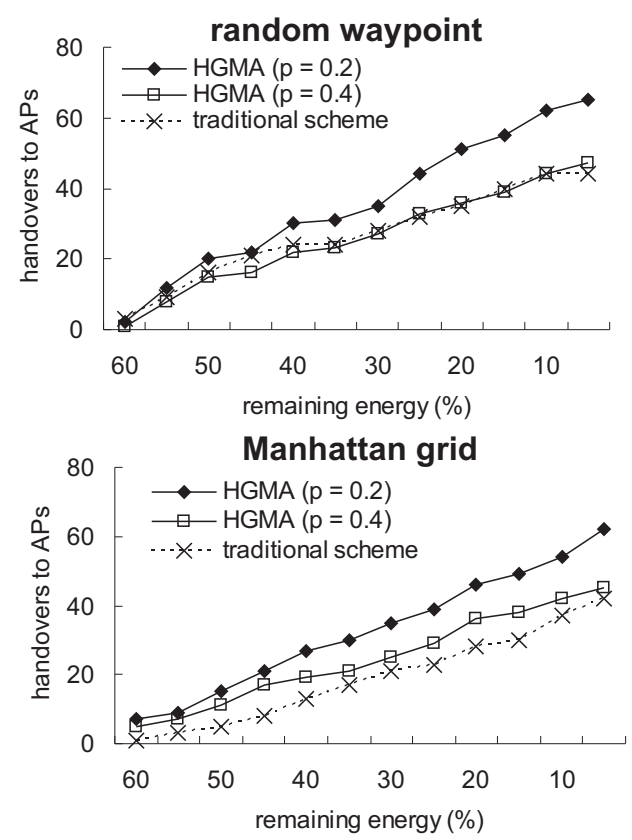

(b) random topology

Fig. 5: Cumulative number of handovers to APs.

TABLE III: Improvement of the number of handovers to APs by HGMA.

\begin{tabular}{ll|ll|l}
\hline $\begin{array}{l}\text { association failure } \\
\text { probability }(p)\end{array}$ & $\begin{array}{l}\text { network } \\
\text { topology }\end{array}$ & $\begin{array}{l}\text { random } \\
\text { waypoint }\end{array}$ & $\begin{array}{l}\text { Manhattan } \\
\text { grid }\end{array}$ & average \\
\hline 0.2 & dense & 1.769 & 1.838 & 1.804 \\
& random & 1.477 & 1.476 & 1.477 \\
\hline 0.4 & dense & 1.231 & 1.270 & 1.251 \\
& random & 1.068 & 1.071 & 1.070 \\
\hline
\end{tabular}

handover scheme. By eliminating unnecessary handovers, reducing the number of network scanning, and avoiding too many interface switching, our HGMA scheme can significantly conserve the energy of MDs related to handover. Simulation results have shown the efficiency and improvement of the proposed scheme under different mobility models of MDs.

\section{ACKNOWLEDGEMENTS}

Y. C. Tseng's research is co-sponsored by MoE ATU Plan, by NSC grants 95-2221-E-009-058-MY3, 96-2218-E-009-004, 96-2219-E-007-008, 97-2221-E-009-142-MY3, and 97-2218E-009-026, by MOEA under grant 94-EC-17-A-04-S1-044, by ITRI, Taiwan, and by III, Taiwan.

\section{REFERENCES}

[1] K. Lu, Y. Qian, H. H. Chen, and S. Fu, "WiMAX networks: from access to service platform," IEEE Network, vol. 22, no. 3, pp. 38-45, 2008.

[2] L. W. Chen, Y. C. Tseng, Y. C. Wang, D. W. Wang, and J. J. Wu, "Exploiting spectral reuse in routing, resource allocation, and scheduling for IEEE 802.16 mesh networks," IEEE Transactions on Vehicular Technology, vol. 58, no. 1, pp. 301-313, 2009.

[3] L. Song, D. Kotz, R. Jain, and X. He, "Evaluating next-cell predictors with extensive Wi-Fi mobility data," IEEE Transactions on Mobile Computing, vol. 5, no. 12, pp. 1633-1649, 2006.

[4] M. Kim and D. Kotz, "Periodic properties of user mobility and accesspoint popularity," Personal and Ubiquitous Computing, vol. 11, no. 6, pp. 465-479, 2007.

[5] M. Afanasyev, T. Chen, G. M. Voelker, and A. C. Snoeren, "Analysis of a mixed-use urban WiFi network: when metropolitan becomes neapolitan," in ACM SIGCOMM Conference on Internet Measurement, 2008, pp. 8598.

[6] M. Ylianttila, M. Pande, J. Makela, and P. Mahonen, "Optimization scheme for mobile users performing vertical handoffs between IEEE 802.11 and GPRS/EDGE networks," in IEEE Global Telecommunications Conference, 2001, pp. 3439-3443.

[7] S. Mohanty, "A new architecture for $3 \mathrm{G}$ and WLAN integration and inter-system handover management," Wireless Network, vol. 12, no. 6, pp. 733-745, 2006.

[8] M. Pischella, F. Lebeugle, and S. B. Jamaa, "UMTS to WLAN handover based on a priori knowledge of the networks," in IEEE International Conference on Communications, vol. 5, 2006, pp. 2009-2013.

[9] F. A. Phiri and M. B. Murthy, "WLAN-GPRS tight coupling based interworking architecture with vertical handoff support," Wireless Personal Communications, vol. 40, no. 2, pp. 137-144, 2007.

[10] P. Yang, H. Deng, and Y. Ma, "Seamless integration of $3 \mathrm{G}$ and 802.11 wireless network," in ACM International Workshop on Mobility Management and Wireless Access, 2007, pp. 60-65.

[11] W. Guan, X. Ling, X. Shen, and D. Zhao, "Handoff trigger table for integrated 3G/WLAN networks," in ACM International Conference on Wireless Communications and Mobile Computing, 2006, pp. 575-580.

[12] J. Nie, J. Wen, Q. Dong, and Z. Zhou, "A seamless handoff in IEEE 802.16a and IEEE 802.11n hybrid networks," in IEEE International Conference on Communications, Circuits and Systems, 2005, pp. 383387.

[13] J. Nie, L. Zeng, and J. Wen, "A bandwidth based adaptive fuzzy logic handoff in IEEE 802.16 and IEEE 802.11 hybrid networks," in IEEE International Conference on Convergence Information Technology, 2007, pp. 24-29.

[14] Y. Choi and S. Choi, "Service charge and energy-aware vertical handoff in integrated IEEE 802.16e/802.11 networks," in IEEE INFOCOM, 2007, pp. 589-597.

[15] J. Nie, X. He, Z. Zhou, and C. Zhao, "Communication with bandwidth optimization in IEEE 802.16 and IEEE 802.11 hybrid networks," in IEEE International Symposium on Communications and Information Technology, 2005, pp. 26-29.

[16] S. F. Yang and J. S. Wu, "Handoff management schemes across hybrid WiMAX $^{\mathrm{TM}}$ and WiFi ${ }^{\mathrm{TM}}$ networks," in IEEE TENCON, 2007.

[17] IEEE 802.16 modules on NS-2 of NIST. [Online]. Available: http://www.antd.nist.gov/seamlessandsecure/download.html

[18] BonnMotion: A mobility scenario generation and analysis tool by University of Bonn. [Online]. Available: http://web.informatik.unibonn.de/IV/Mitarbeiter/dewaal/BonnMotion/ 